\title{
Usefulness of occlusal splints
}

\author{
Jean-Francois CARLIER
}

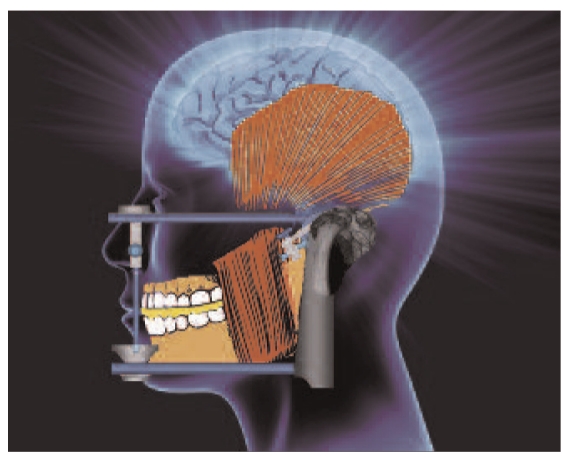

\section{ABSTRACT}

Dentists often use occlusal splints in the management of occlusodontic problems. Because they are considered to provide non-invasive and reversible treatment they are therapeutic devices that conform well to the current philosophy governing protocols for therapy of the masticatory system. Proper design and placement of occlusal splints requires that they respond to precise indications and that practitioners fabricate and maintain them with scrupulous care to assure that patients benefit from them. Splints help practitioners adjust the relationship between the jaws either by suppressing blockage of teeth to relax muscles or by repositioning the condyles in the articular fossas to recapture discs or to reduce painful excess intra-articular pressure derived from degenerative diseases.

Occlusal splints are also suitable as diagnostic tools to validate and evaluate contributions of behavioral, postural, and psychosocial factors to the multifactorial etiology of malfunctions of the masticatory apparatus. The simple introduction into a patient's mouth of an appliance whose benefits have been well described to that patient will serve as a forward step in the psychological management of a TMD problem, especially if bruxism is one of its components.

\section{KEY WORDS}

Occlusal splint,

Inter-maxillary relationship,

Articular repositioning,

Bruxism.

Address for correspondence:

J.-F. CARLIER,

3 , rue LeonTassin,

02880 Bucy-le-Long.

drjfcarlier@orange.fr
Conflicts of interest declared by author: NONE Article received: 09-2011. Accepted for publication: 11-2011. 
Published reviews of the literature frequently remind readers that according to the principles of evidence based medicine there is no justification for treating malfunctions of the masticatory system, temporo-mandibular dysfunction (TMD) with occlusal equilibration or rehabilitation. The absence of EBM documentation demonstrating the effectiveness of specific, and demonstrably reversible, treatment modalities like ortheses is not, however, proof that they are obsolete. Green and Laskin ${ }^{7}$ have shown the importance of understanding the patient's psychosocial environment and family relationships in TMD therapy. The placebo effect, they add, plays a role, pointing out that an orthesis acts, in part, by manifesting the treatment role the practitioner has undertaken. Other, more recent, studies ${ }^{4}$ have validated the relaxing effect of the blocking action of an orthesis.

Even if an occlusal splint is not necessarily indicated as the first step in
TMD therapy, it does constitute a reversible, easy to implement treatment that can validate a diagnostic hypothesis as well as an etiology-based therapeutic device for correction of a temporo-mandibular malady ${ }^{12}$. Before using it, practitioners must complete a complete a clinical examination that suggests a relationship between pathological occlusal relationships and the appearance of TMD or of muscular or articular pain.

The reversible nature of this appliance resides in its innocuous effect on the dental structures that it covers but does not modify and, above all, by the practitioner's scrupulous control of its conception, its fabrication, and the way the patient wears it. A splint can no longer be deemed reversible if it is based on anterior teeth or upon right or left posterior teeth only and is worn for several months because then it will cause noticeable physiological changes of intrusion or extrusion of teeth.

\section{1 - THE MUSCLE RECONDITIONING SPLINT}

A muscle-reconditioning splint becomes indicated as soon as practitioners determine that the pain and malfunctions affecting patients are muscular in origin. They should be designed to suppress occlusal anomalies and to neutralize the alterations of form and position of teeth that cause micro-displacements of the mandible in the approach to maximal intercuspation. These displacements in the position of occlusal equilibrium are caused by asynchronic contractions of the bundles of elevator and other muscles associated with achievement of centric occlusion.

For this reason the splint is made with a flat and smooth surface to avoid creating any obstacle to a patient's finding centric position for the mandible during closure following contraction of the muscles of mastication. It is imperative that the splint be constructed of a material hard enough to make the forces exerted on periodontal mechano-receptors as ubiquitous as possible. The simultaneous 
nature of the contact of the splint's base with opposing teeth and the equality of the occlusal forces thus perceived by the mechanoreceptors instead of what they felt from varied transmission through upper tooth to lower tooth contacts reduce and soothe the activity of the muscles of mastication.

The use of a soft and deformable splint provokes an excess of proprioceptive information from muscles and periodontal tissues that intensify crackling sounds related to munching habits. By multiplying incoherent proprioceptive information soft splints tend to reduce muscular rest periods.

An orthesis employed in TMD treatment should create a situation of anatomic and physiological equilibrium by re-establishing synchronization of muscular contractions, making axial forces symmetrical and well oriented while altering the physiological status of the patient as little as possible.

The appliance should be a $1.25 \mathrm{~mm}$ thermoformed, semi-rigid bite plate covering the occlusal surfaces and extended to the buccal collars of the teeth to insure maximal retention. Its lingual extension should go no further than $5 \mathrm{~mm}$ above the lingual collars of the teeth. These limitations, which improve the splint's esthetics and decrease bulkiness, are designed to increase patient cooperation.

The splint has a flat posterior surface and, if necessary, incorporates a canine-to-canine anterior reinforcement to guide the mandible into a centric position as it closes and to dis-occlude the posterior teeth in excursive movements (fig. 1). Contacts with the teeth of the opposing jaw

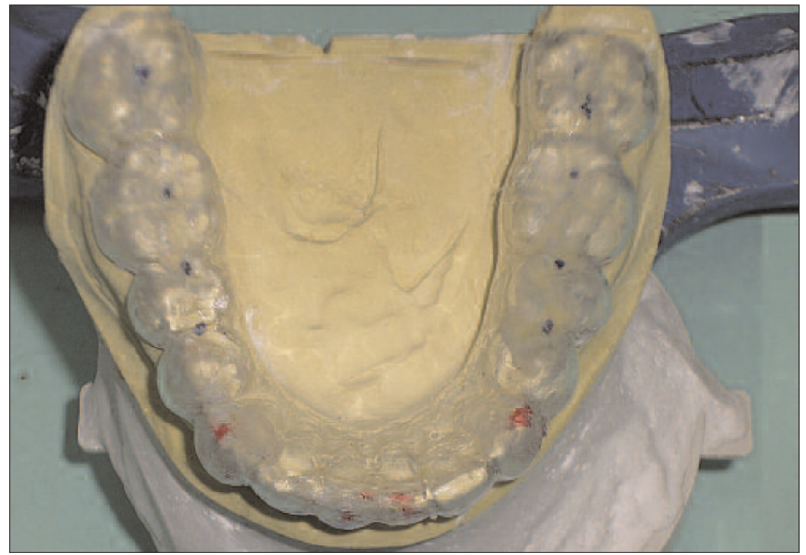

Figure 1

Only the supporting maxillary cusps (blue markings) contact the plastic surface of the splint. The canine guides (in red) assure centric relation.

should be as widespread as possible and only the contact points of the supporting cusps should be preserved to minimize the stimulation of periodontal membranes that provokes muscular hyperactivity.

In choosing which jaw should support the splint, the advantages in comfort of placement on the mandible are this site's lower visibility and its lesser interference with phonation $^{11}$ and the greater access it gives to practitioners in adjusting contact points with direct vision. Practitioners will make their final choice between placement of an orthesis on the maxilla or the mandible by the amount of tooth substance they will have to remove in occlusal equilibration and by the extent of maxillary protrusion so as to limit the invasive nature of the appliance (fig. 2).

The neuromuscular reconditioning splint should be made in the laboratory on models mounted in centric relation and with a slight increase in vertical dimension on a simple articulator. 


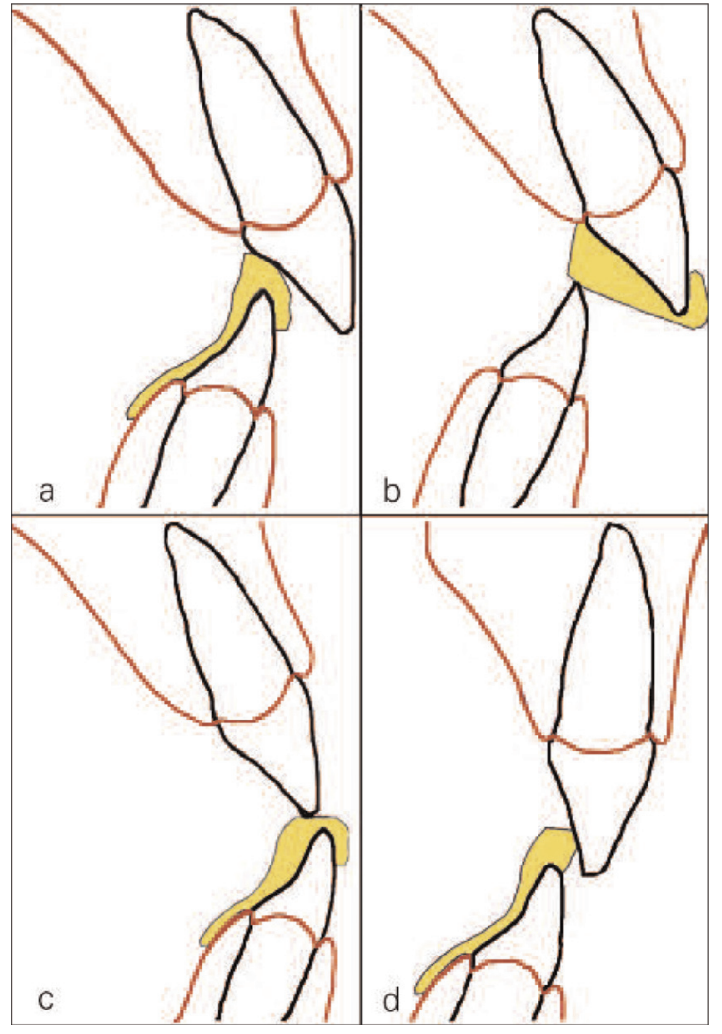

Figures $2 a$ to $2 d$

The extent of maxillary protrusion is a major determinant in decision to place splint on maxilla or on mandible.

The thickness of the splint drawn out in the thermo-formation will, on the average, be $.7 \mathrm{~mm}$, just enough to allow for its final surface to be flattened out. The practitioner or technician can fill in the occlusal irregularities left by the thermo-forming process with photo-polymerizable plastic to optimize occlusal stability (fig. 3).

When placing the splint in the mouth, it is essential for the practitioner to verify its adaptation, its stability, and all tests of comfort for patients that the thermo-forming process suits admirably. The undersurface of a thermoformed device fits far better than plastic plates

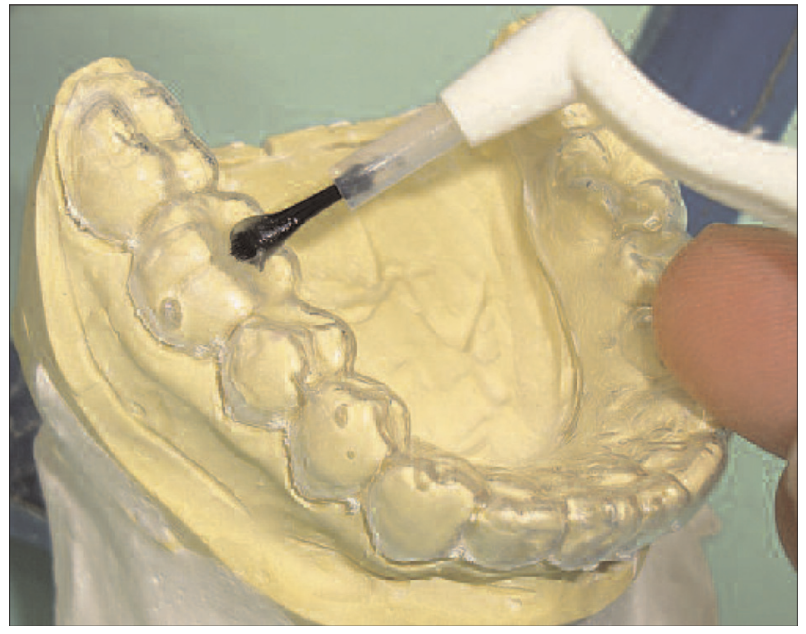

Figure 3

A technician is filling depressions in splint with photopolymerizable plastic to make a smooth surface.

made on a plaster models that are invariably deformed by bubbles or other irregularities, But if a thermoformed splint displays any initial instability, the operator can easily correct this defect by heating its base with a flame and re-seating it in the mouth and having the patient bite down. In this way a defect in the base of a thermo-formed splint when it is first inserted actually helps the practitioner to obtain better retention without giving rise the wedge effect that would result from a similar maneuver with an acrylic plate.

The operator regulates occlusion of the splint by using $40 \mu$ articulating paper to help in spot grinding to generalize cusp contacts when patients, guided by the practitioner's hand, close gently along a physiologically determined arc. If operators try to make these adjustments after patients have closed freely on articulating paper, with no guidance from the dentist, under the voluntary action of the elevator muscles, they will risk reproducing the deviated spatial 
situation of the mandible caused by asynchronic contractions.

TMD splints should be worn primarily at night, but in severe cases it is advisable for patients to wear them in the daytime as well. As mus-

\section{2 - THE ANTERIOR STOP}

On the principle of the Lucia Jig, which was initially designed to register centric relation, an anterior stop is made to constitute a smooth obstacle on the path of a patient's physiological closing ${ }^{1}$, which in disengaging the posterior teeth deprograms afferent proprioceptive messages to periodontal receptors that originate asynchronic muscular contractions. When incisor teeth contact this flat surface, the oral neuro-muscular complex is encouraged to relax, a necessary condition for clinical registration.

One of the principle attractions of this anterior stop is the ease with dentists can construct it in the mouth during an emergency appointment and instantly control pain from crispations of the lateral ptyergoids, and, at the same time confirm the muscular origin of the TMD.

To construct the stop, the practitioner forms a ball of photo-polymerizable plastic (Revotek LC ${ }^{\circledR}, G C$ ) or thermoplastic paste (ISO Functional ${ }^{\circledR}$, $\mathrm{GC})$, places it over the maxillary central incisors, already treated with a separating solution, and covers their incisal edges and their palatal surfaces up to the edge of the hard palate in a layer thick enough to keeping the posterior teeth apart about $1 \mathrm{~mm}$. The practitioner removes the stop from the mouth after it has hardened and grinds it suffi- cular strains begin to disappear, practitioners should make regular adjustments of splints, which, in any case, should not be worn for more than three months.

ciently to reduce contact with mandibular incisors to a single point when patients close on it.

A dentist can make an instant anterior stop simply by cutting a bur box to the width of the two central incisors, filling it with thermoplastic, and holding it in over the incisors until the material sets (fig. 4), thus acquiring the required smooth flat surface with no adjustments needed for its creating the proper amount of posterior disengagement.

The anterior stop, or bite block, is indicated for short-term treatment and for cases with acute symptoms involving both muscles and the TMJ, but dentists must be follow it up with a posterior splint or with occlusal equilibration.

Contra-indications for continuing to wear an anterior stop are strict:

- in cases of disc displacement it will aggravate the disorder by increasing articular compression in the retro-disc area, especially for patients who have not yet received oral behavior counseling on how to reduce muscle crispation;

- for patients whose wearing of the anterior stop has provoked ingression or extrusion of teeth. They should not wear an anterior stop for more than a few days; 


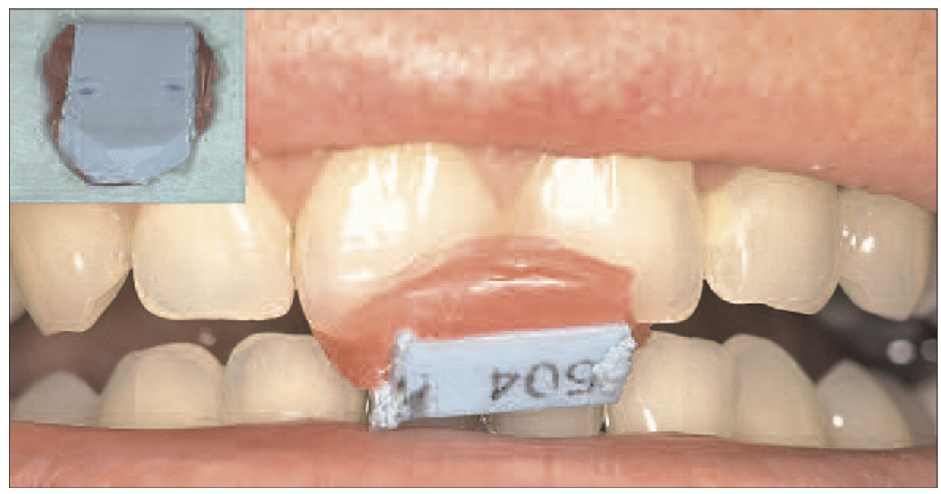

Figure 4

The smooth surface of an anterior stop made with a cut-down bur box makes it available for immediate use with no adjustments.

- when the device is small and not well retained, patients should not risk swallowing it by wearing it at night.

The transitory character of the muscle relaxation obtained ${ }^{2}$, the not negligible risk of aggravating the instability of the condylo-disc complex, and the iatrogenic forces exerted by an anterior splint on the teeth it covers all limit the length of time it can be worn before the dentist registers inter-maxillary relationships and transfers models to an articulator for construction of a posterior muscle reconditioning splint.

\section{3 - THE ARTICULAR REPOSITIONING SPLINT}

Disorders of the masticatory systems are often related to internal derangement of the temporomandibular articulation and the pain associated with it are an indication of the muscular hyper-activity patients have adopted in an unconscious effort to maintain the components of the TMJ in place. An orthesis can help to reposition structures that have been displaced by constraints provoked by trauma or a loss of posterior inter-cuspation. Repositioning splints, also called disc recapturing or anterior repositioning splints, place the mandible in a position that the dentist has determined will re-establish the stabi- lity of the condylo-disc complex, reduce overloading of TMJ structures, and eliminate immobilizing muscular contractions. The dentist makes indentations on the splint surface that will guide the mandible into a new inter-cuspation $^{8}$ that will encourage a therapeutic TMJ relationship (fig. 5).

This splint, made on a base thermoformed over a model of the mandible, will have deep indentations or even a repositioning wall that will oblige the mandible to close into a therapeutic position encouraging disc recapture.

Repositioning splints are indicated in cases of spontaneous reducible 


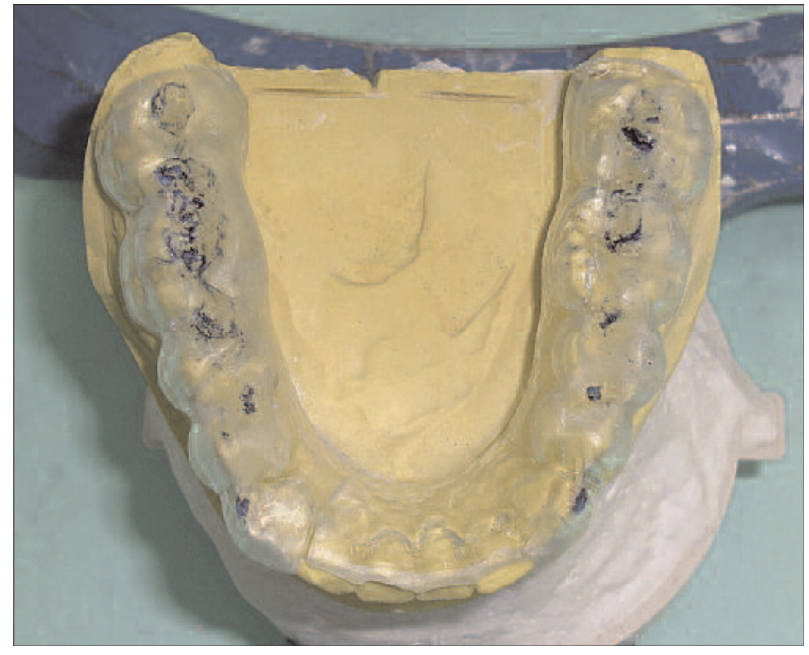

Figure 5

Blue marks show the deep indentations defining the repositioning of the mandible.

disc luxation and are most successful when the clicking sounds of opening and closing occur close to the point of maximal intercuspation.

Dentists can note the limiting zone of disc recapture when clicking is heard on closure, which is why many authors ${ }^{3,5,6}$ recommend discerning the correct therapeutic position for the mandible when it is moving distally.

To accomplish this, dentists ask patients to open their mouths until clicking is heard, then to place the incisors in edge-to-edge position. They then slide their teeth back into maximum occlusion as the mandible is guided by the incisal contact and the discs are held in place by pressure of the heads of the condyles against the anterior slope of the glenoid fossa. The practitioner then determines, at what precise dental position the clicking of return occurs indicating the disc displacement accompanied by a projection click of

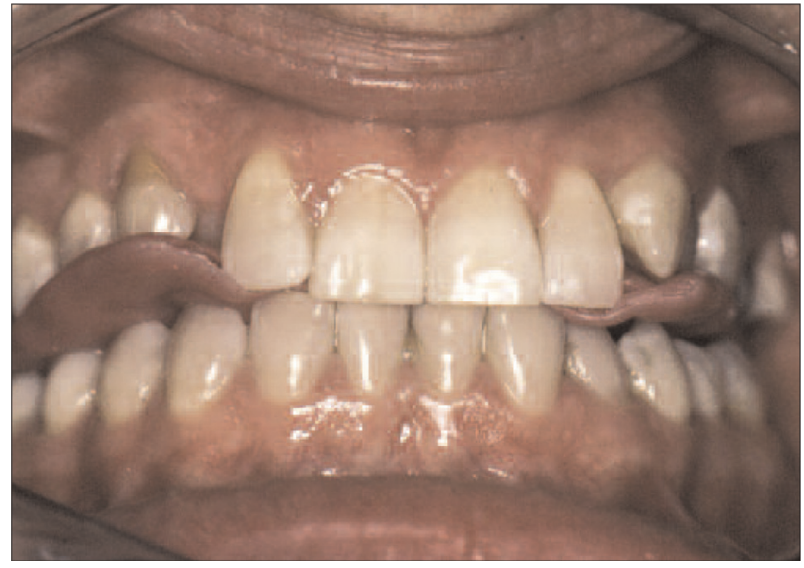

Figure 6

The therapeutic position of disc recapture is registered as the mandible moves distally.

the mandible. After helping the patient make several retraction movements, the dentist registers the correct therapeutic position by taking a three layer posterior hard wax bite Moyco Beauty Pink X (fig. 6) as the anterior teeth are disengaged for the duration of the posterior contact. The interposition of the wax during the mandibular retrusion constitutes a resistance that helps the patient to maintain condylar placement.

The dentist can also determine the point of return clicking by axiographic registrations that indicate on para-condylar recording plates the coordinates of the forward jump that accompanies the luxation. The operator determines the correct therapeutic position on the articulator, ahead of the point of return clicking by blocks placed in the condyle box or by regulating the propulsion screw of the condylar box (fig. 7). The insertion of a $1 \mathrm{~mm}$ thick wedge made by folding the lead paper cover of an intra-oral X-ray film to obtain the necessary decompression for recapture of the disc. 


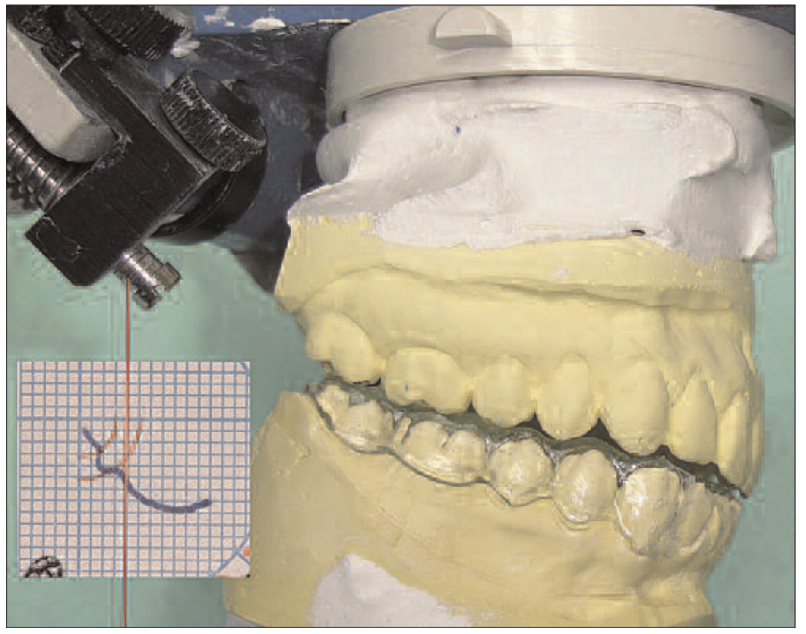

Figure 7

The propulsion screw maintains the head of the condyle in a position in advance of the clicking.

The operator fabricates the mandibular splint on models mounted on an articulator in the therapeutic position provided by the wax bite. It covers the occlusal surfaces of the posterior teeth and its transparent plastic fills the inter-arch space created by the lowering of condyles in the disc recapturing forward position achieved by the wax bite. The indentations in the posterior portion of the splint are about $2 \mathrm{~mm}$ deep and constitute a guidance cone for each antagonistic cusp (fig. 8), encouraging the mandible to enter the therapeutic position as it approaches maximal intercuspation. The anterior teeth are not covered so that contact in propulsive movements will be maintained and so that proprioception in guidance of mandibular movements will be encouraged.

When the splint is placed in the mouth, if the maxillary teeth immediately seek out the indentations in it this validates the accuracy of its ther-

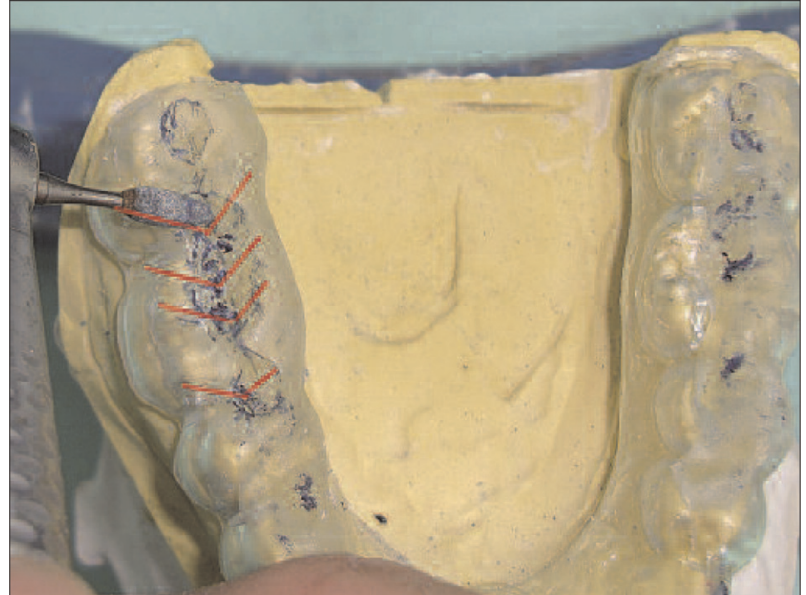

Figure 8

The indentations in the splint constitute a guidance cone for each cusp of the maxillary posterior teeth.

apeutic position. Patients are usually asked to wear repositioning splints at all times except when eating.

In fact it is almost impossible to chew food while wearing splints because the functional schema of mastication cannot be established until the TMJ and muscular problems have been resolved. The indentations are negative imprints of opposing teeth; they are not cusps that can grind up food particles. At best, patients chewing with a splint in place will mutilate it and eventually destroy it; at worst they will generate uncontrollable constraints on oral structures worse than the occlusal disturbance the splint was designed to neutralize.

TMD signs and symptoms begin to dissipate as the splint progressively urges the mandible into a more forward position in accordance with the rules of occlusal equilibration, thus permitting the disc to be recaptured in a less anterior position in the glenoid fossa, while conserving the cooptation of the TMJ components ${ }^{9}$. These 
successive adjustments end up transforming the repositioning splint into a splint stabilizing the new therapeutic

\section{4 - THE DECOMPRESSION SPLINT}

Irreducible disc luxation is characterized by a loss of discal cooptation in maximal intercuspation and by limitation of mouth opening caused by the disc's being tilted forward and inward that provokes a painful stretching of the bilaminary zone.

When an occlusal splint is introduced into the mouth the space between the head of the condyles and the surface of the glenoid increases in an amount equal to the splint's thickness thus providing a decompression of the inflamed TMJ structures.

Technicians can make a simple thermoformed decompression splint on a patient's mandibular model without mounting it on an articulator. The resultant uniform thickness of the device will lower the heads of the condyles and advance the mandible

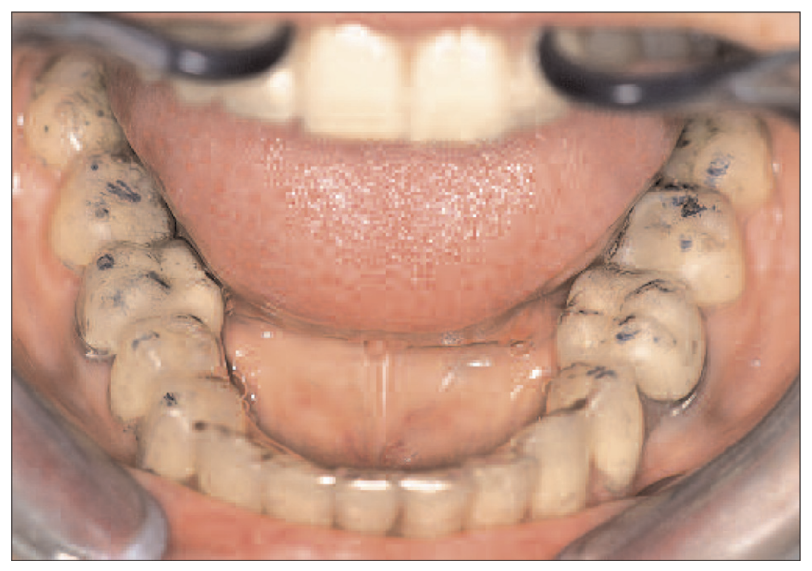

Figure 9

A TMD decompression splint made by adding plastic to the region of teeth 46 and 47 . articular position that accompanies a new occlusion that is mesial to the patient's habitual intercuspation.

slightly after the few moments it takes the patient to adjust to the new biting surface. Dentists can increase the thickness of a splint unilaterally in a selected area to relieve the pain of capsulitis and limit compression caused by edema in the bilaminary zone (fig. 9). Patients are asked to wear the device at all times for a three-week period. As pain decreases in intensity, dentists can begin treatment designed to eliminate the etiological factors. If the patient continues suffer from pain after wearing a decompression splint for several weeks, the dentists should adjust it by adding plastic to the occlusal surface.

In cases of arthritic degeneration, decompression splints can be used

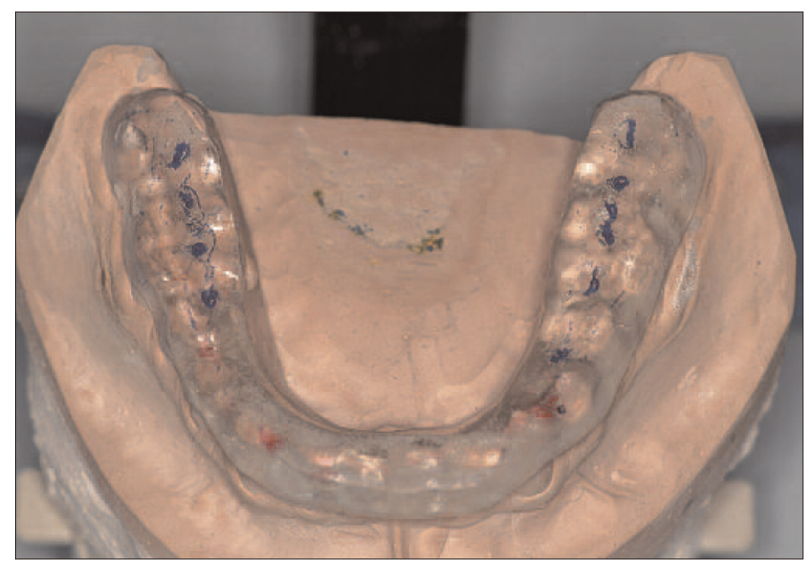

Figure 10

The stabilization splint re-establishes guidance and blockage of the mandible. 
to reduce bone-to-bone contact in the TMJ to allow tissue repair and avoid development of ankylosis. These splints should be made of transparent plastic on models mounted in centric occlusion on articula-

\section{5 - THE STABILIZING SPLINT}

Overall, no matter what its original objective may have been, any interocclusal splint is destined, after progressive adjustments, to become a splint that stabilizes the mandible in the desired reference position that has been obtained.

In this role it establishes a new functional occlusal schema, with structures of guiding, centering, and solidifying cuspation, that can be validated over a period of several months

\section{6 - THE NOCTURNAL PROTECTION SPLINT}

Bruxism is a nocturnal parafunction that, in its abrasion of teeth, diminishes their capacity to guide and stabilize intercuspation and creates wear facettes that exacerbate the condition. We now know that occlusion itself does not cause bruxism and that splints cannot stop it. But they can serve as a protective shields bearing the brunt of erosion in place of the teeth and must be repaired or replaced when they become too thin or too fragile to play a preventive role.

Protective splints take the form stabilization splints made on either arch (fig. 11) of hard plastic covering all the teeth. They are made on models mounted in centric occlusion on an articulator where they are equili- tors. The splint covers the full mandibular arch and carries light indentations to assure stable intermaxillary relationships for several months (fig. 10)

until the dentist decides it is appropriate to undertake definitive stabilization treatment with additive or subtractive occlusal equilibration, with prostheses, or with ortho-dontics.

This stabilization can take the form of wearing full arch nighttime occlusal protective devices for patients who have grinding, clenching, or bruxism habits that erode teeth or imperil prosthetic restorations.

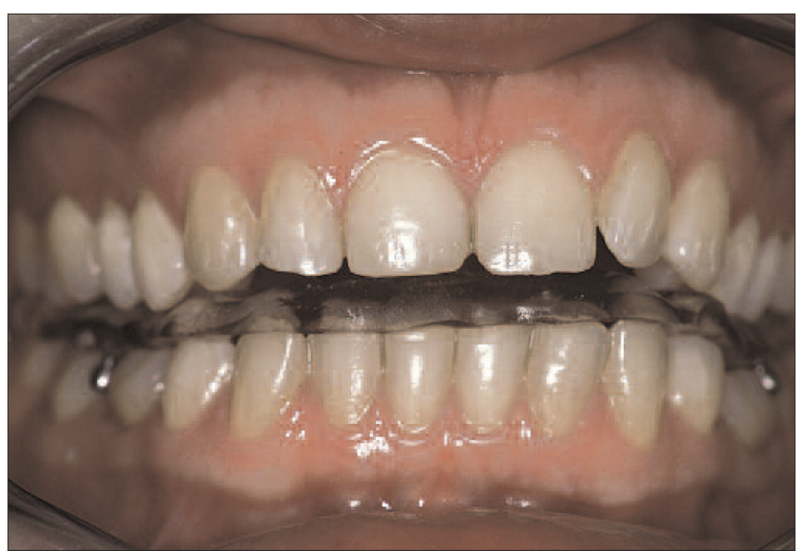

Figure 11

Nocturnal bruxism protective splint.

brated before being delivered to the patient. 


\section{7 - CONCLUSION}

In this article no attempt has been made to present a complete assessment of all the ortheses proposed for treatment of TMD because their number is vast and their numerous authors support their proposals with powerful arguments. But at the end of the day, or night, with regard to bruxism, because these appliances are removable it is the patient who determines their efficacy either because of their unobtrusiveness physically or esthetically, because of the ease of wearing them, and, most important, by the extent of the patients' cooperation with the dentist's therapeutic efforts.

Even though occlusal ortheses are the most popular TMD treatment modality and the easiest one to fabricate and to manage, dentists must take into account two factors that reaching the treatment objective requires: an accurate diagnosis and an effective plan of treatment. And, above all, for effective therapy it is imperative that along with delivering the appliance dentists must provide the patient with a complete and comprehensible explanation of what a temporo-mandibular disorder consists of. And it is equally vital that patients understand what the stakes of this treatment are and what role they play in its execution.

The occlusal splint, in the final analysis, is one element of a global therapy, an instrument, essentially, of patients taking personal charge of their own therapy ${ }^{10}$.

\section{REFERENCES}

1. Abjean J. L'occlusion en pratique clinique. Plømeur : Ed. Bodadeg Ar Sonerion, 2002.

2. Bodéré $C$, Woda A. Effect of a jig on EMG activity in different orofacial pain conditions. Int J Prosthodont. 2008;21(3):253-8.

3. Clark GT Interocclusal appliance therapy. In: Mohl ND, Zarb CA, Carlsson GE, Rugh JD, (eds). A textbook of occlusion. Chicago: Quintessence, 1988:271-84.

4. Ekberg E, Vallon D, Nilner M. The efficacy of appliance therapy in patients with temporomandibular disorders of mainly myogenous origin. A ramdomized, controlled, short-term trial. J Orofac Pain 2003; 17(2):133-9.

5. Farrar WB, McCarty WL. A clinical outline of temporomandibular joint: diagnosis and treatment. Montgomery: Normandy Study group 1982.

6. Gelb H. Clinical management of head, neck and TMJ pain dysfunction. Philadelphia: WB Saunders, 1977.

7. Greene CS, Laskin DM. Splint therapy for the myofascial paindysfunction (MPD) syndrome: a comparative study. J Am Dent Ass 1972;84(3):624-8.

8. Hue O. Manuel d'occlusodontie. Paris : Masson 1992:1307.

9. Okeson JP. Management of temporomandibular disorders and occlusion. St Louis: Mosby Co., 1998.

10. Orthlieb JD, Chossegros C, Cheynet F, Giraudeau A, Mantout B, Perez C, et al. Cadre thérapeutique des Dysfonctionnement de I'Appareil Manducateur (DAM). Inf Dent 2004;39:2626-32.

11. Rozencweig D. Algies et Dysfonctionnements de I'Appareil Manducateur. Paris : CdP, 1994.

12. Unger F, Giumelli B, et Mainetti JL. Dispositifs interocclusaux. Paris : Éditions techniques Encyl Med Chir, France Stomatologie II, 23-446 c-10,1994. 